\title{
SURGICAL TREATMENT IN OVARIAN CANCER - COMPLETE OR OPTIMAL SURGERYP
}

\section{Nițu Teodor Stefan.', Savin Silvia', Costea Daniel Ovidiu', Sârbu Vasile', Șerban Silvia², Nițu Irina²}

${ }^{1}$ Faculty of Medicine, University "Ovidius" of Constanta

${ }^{2}$ County Emergency Clinical Hospital "St. Andrei", Constanta

Teodor Stefan Nitu

Faculty of Medicine, Univeristy ,Ovidius” of Constanta, Universitatii Alee No. 1, Campus B, Constanta, Romania email: nituteodorstefan@yahoo.com phone: +40723988886

\begin{abstract}
Surgical integration in the overall ovarian cancer protocol is conditioned by the performance it has allowed in cytoreduction, the benefits obtained in the interest of the patients in terms of risk (postoperative mortality, severe sequelae, oncology survival criterion at 5 years ).

Surgical treatment remains the fundamental technical means in the treatment of ovarian cancer. It is performed either in the "first intention" (the strand: High-Probability Clinical Diagnosis, Extemporaneous Histopathological Examination Surgery, Continued Intervention, Postoperative Adjuvant Treatments: Chemotherapy, Radiotherapy, Immunotherapy, Hormone Therapy, Second-look 6 Months), or in "second intent" (precise histopathological / pelviscopic or classic diagnosis, followed by chemotherapy, radiotherapy, immunotherapy and radical surgery).
\end{abstract}

Keywords: ovarian cancer, surgical treatment

\section{Introduction:}

Surgical treatment depends on TNM / FIGO pre-therapeutic staging.

a) For stages I and II in developed countries, the approach pathway is more frequent through surgical / operative pelvic surgery / operative laparoscopy.

Stage IA are authors who only indicate unilateral annexectomy, but according to certain conditions (1).

Adjuvant chemotherapy is not necessary in all cases. The second-look laparotomy can be practiced for 6 months all-pelviscopic and addresses patients who apparently have completely responded to chemotherapy or just surgical treatment. It allows an assessment of residual risk and consolidation treatment, directing subsequent attitudes. Most experts in Stage I and II practice the already classical radical intervention: total hysterectomy + bilateral anexectomy + omentectomy (by pubosubombilical or pubo-paraombilical celiotomy) $(1,2)$

b) For Stage III and IV, technical problems are all the more complex as far as hyperbaric interventions are concerned (hysterectomy + bilateral anexectomy + omentectomy is added to large local and visceral extensions) from where the need to study the risk / benefit ratio. 

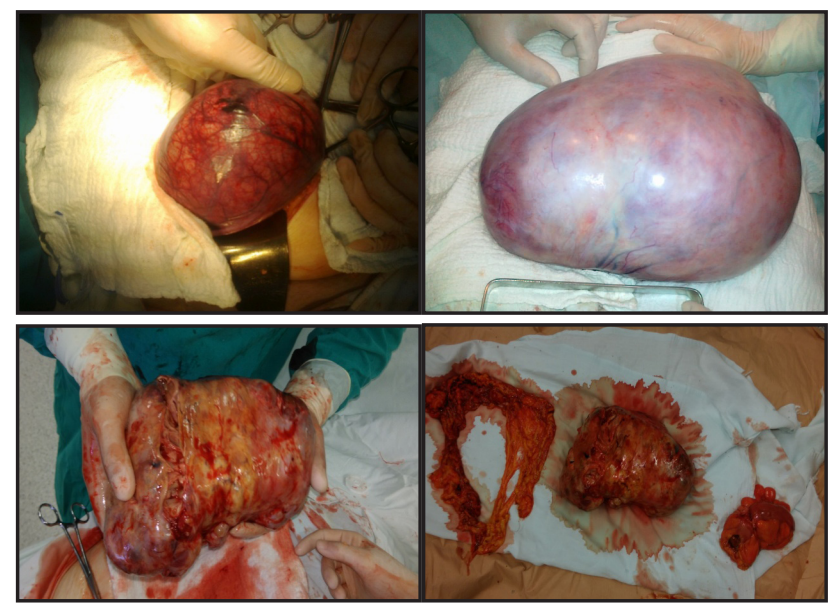

Figure 1 Ovarian malignant tumors - intraoperative aspect (Dr. Niţu Teodor's Archive)

Since chemotherapy has grown more and more into the therapeutic arm of ovarian cancer, and since radiotherapy has perfected its techniques, surgery has simply changed its name from "ultra-aggressive aggressive surgery" to "surgical debulking surgery" (1).

\section{The concept of cytoreduction}

The action of oncostatic agents (chemotherapy, radiotherapy, immunomodulators) is more effective on a cancerous tumor as the volume is lower. There are three reasons for this: bulky tumors are masses where the fraction accessible to treatment is minimal (their growth is pogressive, but the doubling time is reduced, implicitly the reproductive fraction, the less this fraction is the less effective the drugs are ) Cytostatic cells (mutated) or "existing ab initio" appear during cytotoxic administration. These clones develop and occupy more of the tumor mass as long as the leak time is longer and the larger the tumor; bulky tumors are difficult to control through oncostatic treatment due to the increased amount of drug administered (severe side effects on other devices and systems), which is why the administration should be discontinued as well as the tumor tissue of the cancerous tumor to recover between the belts therapeutic. For this reason, the number of therapeutic cures should be higher as the tumor is bulkier. "Surgical cytoreduction" means surgery to reduce the tumor volume before or after cytotoxic attack (it is a "shrinking" surgery) to reduce "nothing" or "almost nothing".

\section{Coding of cytoreduction operations}

It's a difficult thing, but many distinguished authors - optimal surgery (leaves no visible macroscopic tumor residue); - almost optimal surgery (leaves residues not exceeding 1.5 $\mathrm{cm}$ diameter); - Suboptimal surgery (leaves voluminous residue). In stages I and II: tumor proliferation is limited to ovaries or pelvic structures. Standard surgery is total hysterectomy with bilateral anexectomy + omentectomy + peritonectomy (exit of the peritoneum, Douglas and vesicouterin sac assy). In stages III and IV: extension above the upper pelvic strain to the large peritoneal cavity. Unfortunately, these severity extremes are the most numerous in practice $(60-80 \%)$. Optimal reduction can only be achieved at the expense of large, very laborious extensions. Indeed, it is less common that full hysterectomy with bilateral anexectomy, omectomy, and peritoneal resection, more or less extensive, give the expected results (stage IA). In most cases digestive digestion is required: recto-sigmoid resection (segment of the most direct and frequently threatened digestive tract); - ileo-cecal resection (more or less extended to other segments of the colon - hemicolectomy); more or less stretched resection of the mesentery and the small intestine; - part or all of a certain viscera (spleen, stomach, liver, etc.) These cases are regrouped in stage IIIb, and the surgery that reduces them is undoubtedly an "ultra-aggressive surgery".

\section{The problems of cytoreduction surgery}

Stages I and II. As discussed at Stage IA (reaching a single ovary), some authors only indicate unilateral anexectomia, depending on certain "conditions". However, most clinicians practice after the extemporaneous histological examination of the piece, total hysterectomy + bilateral anexectomy + omentectomy + eventually peritonectomy. In the classical conception the surgical approach is done only by pubo-ombilical or pubo-paraombilical medial celitotomy. It allows the complete exploration of peritoneal cavitation and authorizes, after viewing, the extension of surgery from simple anexectomy and histologically extemporaneous examination to radical or ultra-radical surgery (celitotomy 
may prolong pubo-xifoidian). In this regard, there are authors who contraindicate Phannenstill or Maylard laparotomy / celitotomy transversal in any surgical treatment of any tumor of the ovary. In some cases, the same author does not practice ostectomy in the first intention, considering the large epiplone as the most important "trapper" of neoplastic cells in ovarian cancer, extirpating it necessarily on "second-look celiotomy". In the modern concept, the surgical approach is made per-pelviscopic especially when it comes to young women. Pelviscopy allows for an exploration at least as complete as the classic middleiatomy. If the ovary tumor is well located in the peritoneal cavity and all signs lead to Stage IA, some authors may practice conservative extirpation by Phannenstill transient celiotomy (1), but only the classical middleiotomy.

Stages III and IV. The technical aspects are numerous and serious:

- the great epiploon and the parietal peritoneum. When these areas are the only ones caught in the extraovarian extension, omentectomy and peritonectomy (the most common of Douglas and vesicouterine) are practiced; interventions are easy to apply and non-dangerous;

- the recto-sigmoid junction. Extirpation does not raise particular problems when the recto-sigmoid junction is the only part of the digestive system requiring resection. Expansion into "block" is possible immediately followed by surgical restoration of digestive continuity (postoperative morbidity is reduced).

- other segments of the colon, mesentery and small intestine. Problems begin when colectomy (straight hemicolectomy that includes the ileo-cecal junction) and extra, more or less extensions of the mesentery and the small intestine. Postoperative morbidity is frequent and severe. Postoperative sequelae, starting from a certain limit, are severe and often difficult to tolerate.

- supramezolic viscera. Situations become insoluble when it is necessary to exert certain supramezolic viscera (stomach, spleen, liver, etc.). The risks are immense.

Stage IV. Loco-regional sprains (ultraintervention) whatever their extensibility are illogical. Ovarian cancer is already a general illness by remote microdisignation. The question that arises in essence is the place of surgery (useless, deadly), the complementary treatments, the adjuvants being the only accepted solutions, with other palaetic effects $(1,2)$.

\section{The place of cytoreduction surgery}

Surgical integration in the overall ovarian cancer protocol is conditioned by the performance it has allowed in cytoreduction, the benefits obtained in the interest of the patients in terms of risk (postoperative mortality, severe sequelae, oncology survival criterion at 5 years ). Stages I and II. Cytoreduction is optimal for an acceptable "price" (total hysterectomy with bilateral anexectomy + omentectomy + peritonectomy). In particular, for Stage IA the surgical risk is almost null, and the woman's desire to maternity can be achieved (in cases where they meet the conditions for unilateral annexectomy). A number of authors consider the secondary, postoperative adjuvant chemotherapy to be required. Recent studies demonstrate the need for adjuvant chemotherapy in IC and IIC stages (positive peritoneal cytology and tumor grading II and III) (3) Stages III and IV. Optimal or almost optimal cytoreduction can only be achieved at the cost of difficult or even inadmissible sacrifices $(86,88)$. The first question, then, is whether it deserves to be or not to be tried. The second question: Whether it is worthwhile, when it is better to be practiced: before or after chemotherapy (and especially after cure chemotherapy $-3,6,9, \ldots$ ). Griffiths (Boston), Joyeux (Montpellier) found in second-look control laparotomies that prolonged survival is more common in women undergoing ultrasound surgery at first intention. According to the same authors, second-degree cytoreduction (after 6, 9 cure chemotherapy) does not enjoy the same results, even having a bad reputation. There are also authors who do not share this view: they support chemotherapy in the first intention (transforms tumors initially inoperable into operable tumors), the results obtained after the ultrasoundal second-order cytoreduction surgery are at least equivalent to those obtained after the ultraagressive surgery of the first intention $(4,5)$. 

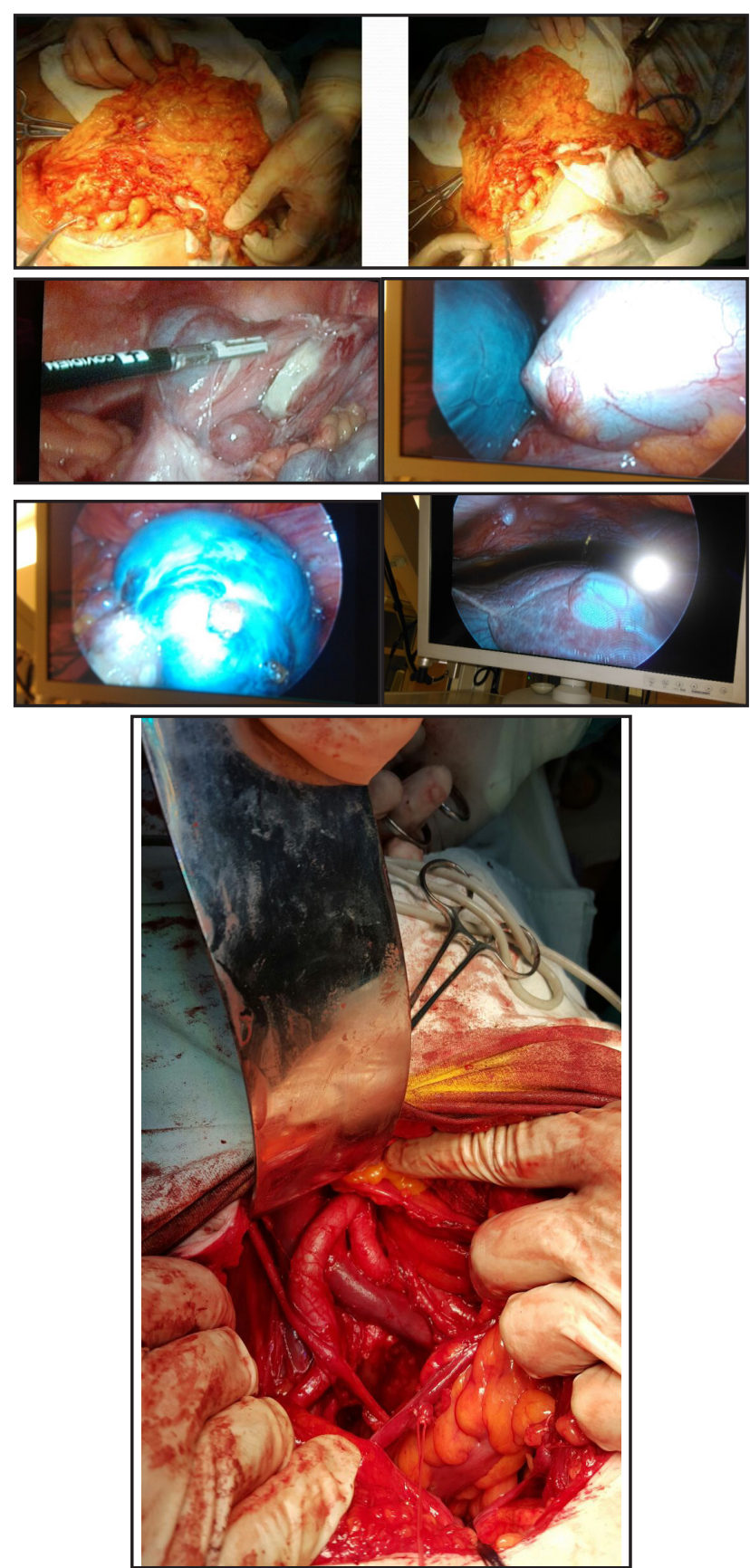

Figure 2 Intraoperative Aspects in Ovarian Cancer (Dr. Niţu Teodor's Archive)

In terms of ultrasound second-line surgery, there is the question of the optimal number of chemotherapy cures: after long-lasting (6-9) treatment the patients are ill and have poor prognosis, second-order cytoreduction aggravating the progression; after short courses (induction chemotherapy / 2-3 cures) a number of cases respond favorably, second-line surgery with a relatively favorable prognosis (more remissions, longer lasting survival) (1).

\section{Laparotomy / celiotomy "second-} look" (Wangestein, 1940) - This intervention, contrary to expectations, addresses patients who apparently have completely responded to chemotherapy or cytoreduction surgery (almost optimal) and who want to check the quality of this response. It is not addressed to those patients who, after a suboptimal initial chemotherapy and consecutive chemotherapy, have responded partially, attempting by a second effort (most often unnecessary) to remove what was not proposed and / or failed at first intervention. The opposition between second-look laparotomy and secondary debulking is actually much more tense than it seems. Macroscopic lesions are found on average on 1 case of 2 patients who appear to be cured after initial chemotherapy. The problem in such cases is the opportunity for secondary cytoreduction, reinterveation of exertion. The second-look laparotomy in a case of 3 does not show residual lesions either at inspection (direct viewing) or at palpation. The multiple samplings that are used on this occasion (this means the retroperitoneal space, respectively, the lomboaortic and pelvic lymph nodes) show instead microscopic cancerous lesions in a case of three. Chemotherapy in these women should be continued (in the same formula or other formulas), with the benefits being noted in over $50 \%$ of cases. In this regard, it should be noted that those women whose second-look control is absolutely negative (cytological and histopathological) are not relieved of relapse, and $40 \%$ of cases recur. All the more so in these situations is the "cytostatic treatment of consolidation". Finally, considering these observations, the question arises as to whether the second-look laparotomy is beneficial, knowing that the risk of recurrences remains elevated even when the multiple lesions are negative on the histopathological examination.

According to some authors, on comparative studies between groups of women who have accepted second-look laparatomy and others who have refused, there is no difference (on the basis of oncology survival at 5 years). Chemotherapy is essential in all cases, regardless of whether or not the second-look laparotomy is practiced $(1,3,6)$. The same treatment and clinical-biological surveillance rules are imposed in one case and 
the other, having a practical questionable utility since it provides information that is already anticipated. With regard to second debulking, even with the presence of macroscopically obvious residues, the results are doubtful because the risks of relapse are increased even in women without residues and with multiple negative sampling on the microscopic examination (1)

\section{Diagnostic and operative pelviscopy}

It has been used as a particularly useful method in the "second-look" manner. He was later criticized for not being able to fully evaluate pelviabdominal cavity after initial intervention due to adhesions, newly created artificial septa between different compartments, impossibility to explore retroperitoneal space in other situations. In the surgical treatment of ovarian cancer, "debulking surgery" is the most appropriate name (6). When surgical cytoreduction is optimal, the results are good. In cases where surgery is almost optimal (does not leave tumor residues greater than $15 \mathrm{~mm}$.), Oncological survival is almost twice as long as in cases where suboptimal surgery was practiced. Optimal surgery is the only one that provides rigorous clinical and statistical data for appreciable chances of prolonged survival. Of course, the question is whether it is worthwhile for the future to perform ultra-rigorous surgery with the major risks they are having, knowing that these complex operations never guarantee the complete removal of ovarian cancer in advanced stages.

\section{The importance of complete surgery and criticism of extensive cytoreduction surgery}

The current one speaks of complete cytotoxicity rather than optimal, the first author to suggest this was Eisenkop, who suggested that it should be the goal of debulking surgery. $\mathrm{He}$ conducted a study published in 1998, which encouraged extensive surgery in the upper abdomen, including diaphragmatic resections and diaphragmatic peritonectomy, hepatobiliopancretic resections, splenectomies, demonstrating the usefulness of extrapulpine surgery (7). If cytoreductive surgery was accepted for pelvic lesions, in the case of extrinsic lesions, the opinions were divided. Starting with Stage $1 \mathrm{C}$, which accounts for approximately $67 \%$ of diagnosed ovarian neoplasms, patients have secondary determinations in the upper abdomen, requiring extensive resections to maximize the debulking effort. This has led to the conclusion of the opponents of the idea of debulking in the upper abdomen, that the biological aggression of the tumor will dictate the subsequent evolution of the patient, the extensive cytoreduction surgery not being able to induce a survival benefit $(8,9)$.

\section{Conclusions}

Lately, the idea of maximal surgical debulking has been implemented, which makes extralpine resections (in the upper abdomen, thoracic and even neurosurgical) to become a component of the cytoreductive surgery, of course in specialized centers with a large volume of interventions (10) . Excessive mutilation is a biologically "expensive" price paid by any woman in such circumstances, known to be the perfection of the disease and not to forget the quality of life of these patients after these laborious interventions.

Thus, the increase in the chance of survival and implicitly the control of ovarian cancer is done only through measures of early detection of the high risk groups after the age of 30 years (women who in the heredo-collaterals have genital or maternal breast cancer, women without pregnancies, with benign tumors in the past, with endocrine pathology or other neoplasias).

\section{Refecences:}

1. Dargent D., Principles and practice of gynecologic oncology. Lippincot Williams\&Wilkins, 1992; 269-289; 381-463

2. Piver MS. Ovarian Malignancies: Diagnostic and Therapeutic Advances: Churchill Livingstone; 1987, pp 245-297

3. Dorval T., Cytokines and Cytokine Receptors. Physiology and Pathological Disorders. Taylor\&Francis e-Library, 2005 ISBN 0-203-30513-2.

4. DiSaia PJ, Creasman WT. Clinical Gynecologic Oncology E-Book: Elsevier Health Sciences; 2012.

5. Nygel Acheson, Luesley D., Gynaecological Oncology, MRCOG 1988, ISBN 978-1- 
906985-21-9.

6. Hoskins W.J.,, Carlos A. Perez, C. A., Young R.C., Barakat R., Markman M., Randall M.,Gynecologic Oncology Lippincott Williams\&Wilkins, 2005, ISBN 0-78174689-2.

7. Eisenkop SM, Friedman RL, Wang HJ. Complete cytoreductive surgery is feasible and maximizes survival in patients with advanced epithelial ovarian cancer: a prospective study. Gynecologic oncology. 1998 May 1;69(2):103-8.

8. Horowitz NS, Miller A, Rungruang B, Richard SD, Rodriguez N, Bookman MA, Hamilton CA, Krivak TC, Maxwell GL. Does aggressive surgery improve outcomes? Interaction between preoperative disease burden and complex surgery in patients with advanced-stage ovarian cancer: an analysis of GOG 182. Journal of Clinical Oncology. 2015 Mar 10;33(8):937.
9. Crawford SC, Vasey PA, Paul J, Hay A, Davis JA, Kaye SB. Does aggressive surgery only benefit patients with less advanced ovarian cancer? Results from an international comparison within the SCOTROC-1 Trial. Journal of clinical oncology. 2005 Dec 1;23(34):8802-11..

10. Suciu N. Bacalbasa N, Achimas Cadariu P, Blidaru A: Treatise of extrapelvic surgery in ovarian cancer. Ed Academiei Romane: 1425,2017 